\title{
Global and regional health emergencies dominate discussions at the World Health Assembly and Regional Committee 2015
}

\author{
Ala Alwan ${ }^{7}$
}

The World Health Assembly, held in May each year, is traditionally an opportunity for Member States to review WHO's work over the past year and to agree on the priorities and strategies for improving global health in the years ahead. This year the Health Assembly was much the same in format, but the discussions were more intense, more in depth and more critical of WHO than they have been for many years.

In the past year, we have seen a series of large-scale global health emergencies, across the Region and across the world. Currently WHO, together with the United Nations and other partners, is simultaneously tackling multiple highlevel emergencies, including five at the highest level, as well as several other emergency situations of concern. The outbreak of Ebola virus disease in West Africa stretched WHO's resources to the limits, while the scale of population movement and humanitarian crisis within a number of regions, including our own, has been unprecedented. Discussion at the Health Assembly was dominated by the impact and implications of such emergencies, both in specific discussions on WHO's response to large-scale emergencies, Ebola and the International Health Regulations, and in discussions on WHO reform and its proposed programme budget for 2016-2017.

Among the documents the Assembly considered was the report of the Ebola Interim Assessment Panel, which was requested by the WHO Executive
Board at its special session on Ebola in January this year. In its report, the Panel highlighted the fact that WHO consists not only of the Secretariat but also the Member States and noted, "Together, the WHO leadership and the Member States need to take determined action to address the challenges at hand". It became very clear during the Health Assembly that these challenges are being taken extremely seriously. In response, action is being taken by WHO to strengthen outbreak and emergency response work. In our Region we have restructured this area of work and considerably scaled up in both the Regional Office and countries but much needs to done to respond to the increasing demands in the Region heavily affected by crisis. A US $\$ 100$ million contingency fund will be set up at the global level to provide financing for field operations for the first 3 months of an emergency. Member States were supportive of WHO's essential role in leading and responding to global health emergencies and, while critical of aspects of its performance, appreciated that the Organization must be properly resourced and enabled to do the work expected of it. Later this year, Member States will meet again in a "financing dialogue" to discuss how the contingency fund and programme budget for 2016-2017 should be financed.

A number of other crucial issues for global health were discussed. The Health Assembly adopted a global action plan on antimicrobial resistance and urged Member States to implement it, while adapting it to their national priorities and specific contexts, and to mobilize resources accordingly. The plan requires Member States to have in place by 2017 national plans that will cover the use of antimicrobial medicines in both human health and animal health. This is a vital issue that no country can ignore. Later this year Oman will host, in collaboration with $\mathrm{WHO}$, a highlevel meeting of ministers of health and agriculture, to which all Member States of the Region have been invited.

The Health Assembly endorsed a resolution to improve access to sustainable supplies of affordable vaccines. This is a key issue for low- and middleincome countries, including those in our Region, aiming to extend immunization to the entire population, and particularly with the new generation of vaccines now available. Countries of our Region were instrumental in supporting the resolution tabled by Libya on this issue and on moving forward in implementing the global vaccine action plan endorsed in 2012. The Assembly also endorsed a global malaria strategy aimed at reducing the global disease burden by $40 \%$ by 2020 and by $90 \%$ by 2030 . The strategy provides a comprehensive framework for countries to develop their own tailored programmes to achieve these targets.

The Health Assembly endorsed its first ever resolution on the health impacts of air pollution. The issue was intensely debated with many competing concerns. Nevertheless, in a show 
of unity, Member States came together to address an issue that is among the leading causes of disease and death, and the world largest single environmental health risk. Among other things, the resolution highlights the key role national health authorities need to play in raising awareness about the health impacts of air pollution, the need for cooperation between different sectors and integration of health concerns into all air pollution-related policies, and the need for improved morbidity and mortality surveillance of all illnesses related to air pollution.
Next month Ministers of Health of the Region gather again at the annual meeting of the WHO's regional governing body, the Regional Committee. There will be a special session highlighting, among other things, emergencies and humanitarian action in the Region and implementation of the International Health Regulations 2005. Both of these are core issues in relation to WHO's work in the Region, and to each other. Conflict and the humanitarian consequences continue across the Region, and now increasingly beyond it. We will be putting before Member States the action we have taken with partners in the past year to support communities and displaced populations, as well as the strategic steps we have taken to support the Region as a whole, including the establishment of a regional solidarity fund, enhancement of regional capacity and a strategy for monitoring and assessing implementation of the International Health Regulations. In turn, we will be looking to our Member States to endorse the course we are following and to guide us further on the way forward. 